\title{
Éditorial
}

\section{Appelons un chat un chat}

Il est des moments où il est bon de rappeler la véritable signification des mots ; plusieurs événements de différente importance me poussent à faire le point sur le bon et le mauvais usage de certains mots dans notre profession. Je ne suis pas sûr que les quelques exemples qui vont suivre soient exhaustifs, mais ils sont pour le moins significatifs.

En premier, et ceux qui me connaissent ne seront pas surpris, employons correctement les mots radionucléide et radioélément. Le premier est un isotope radioactif, ${ }^{60} \mathrm{Co}$ par exemple, le second est un élément chimique, l'uranium par exemple dont tous les isotopes sont radioactifs. Le cobalt-60 n'est pas un radioélément, pas plus que l'uranium-238, tous deux sont des radionucléides. C'est l'uranium qui est un radioélément.

Le deuxième mauvais usage des mots que l'on rencontre encore trop souvent dans notre profession est l'emploi à contre sens du sievert. Dans ses nouvelles recommandations à paraître, la CIPR est beaucoup plus claire que dans sa publication 60 qui prônait le pardon. Le sievert, tel qu'il est conçu, ne peut être utilisé que pour des effets différés, stochastiques, il ne doit en aucun cas se substituer au gray pour les effets déterministes précoces, et pourtant, de nombreuses échelles de risques nous indiquent des risques aigus exprimés en sievert.

Le troisième exemple sort d'un texte officiel, et là, cela devient encore plus grave, mais ce n'est en aucun cas, une excuse pour ne pas réagir. En effet, j'ai lu récemment «intensité de rayonnement» exprimée en sievert. La confusion est énorme, un rayonnement n'est pas une dose, il faut corriger ces textes officiels rapidement car sinon la législation deviendra ambiguë et porte ouverte à contestation.

Enfin, pour clore aujourd'hui cette liste, la dramatique affaire Livitienko nous a brutalement rappelé qu'il y avait une relation fondamentale inverse entre la période radioactive et l'activité spécifique. Alors, pourquoi voir partout pour les déchets l'association des mots « haute activité » et «vie longue ». C'est l'un ou l'autre. On a pu tuer sans problème avec le ${ }^{210} \mathrm{Po}$ dont la période est de 138 jours, puisque seuls quelques microgrammes suffisaient; faites le calcul, cela aurait été bien difficile et même impossible avec du ${ }^{239} \mathrm{Pu}$ dont la période est de 24000 ans.

DOI: $10.1051 /$ radiopro:2007003

RADIOPROTECTION - VOL. 42 - $\mathrm{N}^{\circ} 1$ (2007) 
ÉDITORIAL

Pire encore, pour reproduire les mêmes effets avec du ${ }^{237} \mathrm{~Np}$, ce n'est pas le contenu d'une tasse de thé qu'il fallût mais toute l'eau d'une piscine.

Comme vous le voyez, le mauvais usage des mots peut aller de l'anecdote à des choses plus dramatiques. Il y a sûrement d'autres coquilles qu'il nous faut corriger. $\mathrm{Au}$ moment où une nouvelle édition d'un dictionnaire nucléaire est à l'ordre du jour, c'est le moment de signaler tous les errements pour, espérons-le, qu'ils cessent.

Sinon, bonne lecture de ce nouveau numéro, qui, je l'espère, est sans coquille.

Henri Métivier Président du comité de la revue 